\title{
Dynamics of linguistic opinion formation in bounded confidence model
}

\author{
Yucheng Dong, Xia Chen, Haiming Liang* , Cong-Cong Li \\ Business School, Sichuan University, Chengdu, 610065, China
}

\begin{abstract}
Opinion dynamics is a fusion process of individual opinions based on the established fusion rules. The existing opinion dynamics models assume that agents express and receive their opinions in a numerical way. In this paper, we focus on the opinion formation in linguistic environment (i.e., linguistic opinion formation), and propose a linguistic opinion dynamics (LOD) model in the framework of the bounded confidence and the 2-tuples linguistic model with numerical scales. In the LOD model, agents express and receive the opinions by using the simple terms in a linguistic term set with finite granularity at each time. Based on the LOD model, we present some theoretical analyses to reveal the conditions to form a consensus or splits in the linguistic opinion formation. Furthermore, we design some simulations to investigate the effects of the bounded confidence and the uniformities of linguistic term sets on the linguistic opinion formation. The simulation results show that: (i) with the increase in the bounded confidence and the uniformities of linguistic term sets, the opportunity for reaching a consensus will increase, and the time for reaching a consensus will become shorter in the dynamics of linguistic opinion formation; (ii) there exists a critical point in the evolution of linguistic opinions, which is called agreed confidence in this paper, and a consensus among the agents will be reached if the value of the bounded confidence is equal or greater than the agreed confidence.
\end{abstract}

Keywords: Opinion dynamics; Bounded confidence; 2-tuples linguistic model; Linguistic opinions; Fusion process

\section{Introduction}

Opinion dynamics is widely used to model the diffusion, fusion and evolution of opinions expressed by a population of interaction agents. There are two varieties of stabilized patterns in opinion dynamics, i.e., consensus or splits. To discuss the issues regarding stabilized patterns, many opinion dynamics models had been proposed. An early formulation of such a model was given by French [1]. Following that, some interesting opinion dynamics models with different fusion rules had been proposed to discuss the stabilized patterns, such as the voter model [2, 3], the De Groot model [4], the persuasiveness and supportiveness model [5], the Alexford model [6], and the bounded confidence model (e.g., [7-14]).

Recently, the bounded confidence models in opinion dynamics have received significant attentions. In the bounded confidence models, each agent only considers those agents whose opinions differ from his/her own not more than a certain confidence level. The Hegselmann and

\footnotetext{
* Corresponding author. Tel.:+86 2882673492
}

E-mail address: hmliang@scu.edu.cn. 
Krause (HK) model [7-8, 10-12] and the Deffuant, Weisbuch and others (DW) model [9, 13-14] are two classical bounded confidence models, which both rely on the idea of repeated averaging under bounded confidence. However, they differ in the fusion rules. In the DW model, agents meet in random pairwise encounters after which they compromise or not. In the HK model, each agent moves to the average opinion of all agents which lies in his/her area of confidence. On the basis of the original DW and HK models, different types of extended research works have been proposed: multidimensional opinions [15-17], heterogeneous bounded confidence [18, 19], social networks [20-22], communication regimes [23-25], and opinion control [26-28].

In this paper, we discuss the opinion dynamics in linguistic environment and propose the linguistic opinion dynamics (LOD) model, which are motivated by the following aspects:

(i) The classical opinion dynamics models mentioned above are all proposed in numeric environment. However, in the real-world opinion dynamics situations, agents often express and receive opinions linguistically (i.e., linguistic terms). The use of linguistic terms implies the need for computing with words (CWW) [29-36].

(ii) Generally, the classical opinion dynamics models assume that agents express the numeric opinions in a continuous interval (e.g., $[0,1]$ ). However, since the human languages usually cover only a limited number of linguistic terms, then the linguistic opinions expressed and received by agents are simple terms in the linguistic term sets with finite granularities. The continuous (or 2-tuple) linguistic opinions only appear in operations.

The proposal can be applied to address some practical linguistic opinion formation problems. For example, when the citizens express their opinions using the simple linguistic terms (e.g., very unfavorable, somewhat unfavorable, neutral, somewhat favorable and very favorable) on the published health policy, our proposal can provide a decision support for the government to analyze the dynamics of public linguistic opinions.

The rest of this paper is organized as follows. We provide the basic knowledge regarding the 2-tuples linguistic representation model, the numerical scale model and the original HK model in Section 2. Section 3 proposes the linguistic opinion dynamics problem and constructs the LOD model. Subsequently, we present several theoretical results of the LOD model in Section 4. We give an extensive exploration of the LOD model by a series of simulation experiments in Section 5. Finally, Section 6 concludes the study.

\section{Preliminary}

This section introduces some basic knowledge regarding the 2-tuples linguistic representation model, the numerical scale model and the HK model, which will provide a basis of this paper.

\subsection{The 2-tuples linguistic representation model}

The basic notations and operational laws of linguistic variables are introduced in [37]. Let $S=\left\{s_{j} \mid j=0,1, \ldots, G\right\}$ be a linguistic term set with odd granularity $G+1$, where the term $s_{j}$ 
represents a possible value for a linguistic variable, and it is required that the linguistic term set should satisfy the following characteristics:

(i) The set is ordered: $s_{i} \leq s_{j}$ if and only if $i \leq j$;

(ii) There is a negation operator: $\operatorname{Neg}\left(s_{i}\right)=s_{j}$ such that $j=G-i$.

Herrera and Martínez [37] initiated the 2-tuples linguistic representation model. Let $S$ be a linguistic term set with the granularity $G+1$. Then, the 2 -tuples that expresses the equivalent information to $\beta$ is obtained with the following function: $\Delta:[0, G] \rightarrow S \times[-0.5,0.5)$, where

$$
\Delta(\beta)=\left(s_{j}, \alpha\right), \text { with }\left\{\begin{array}{c}
s_{j}, j=\operatorname{round}(\beta) \\
\alpha=\beta-j, \alpha \in[-0.5,0.5)
\end{array}\right.
$$

Clearly, $\Delta$ is a one to one mapping function. For convenience, its range is denoted as $\bar{S}$. Then, $\Delta$ has an inverse function with $\Delta^{-1}: \bar{S} \rightarrow[0, G]$ with $\Delta^{-1}\left(s_{j}, \alpha\right)=j+\alpha$. Specially, if $\alpha=0$, then the 2-tuples $\left(s_{j}, \alpha\right)$ is reduced to a simple term $s_{j}$.

To compare the 2-tuples information, the 2-tuples linguistic comparison operator has been presented in [37]. Take any 2-tuples $\left(s_{k}, \alpha\right)$ and $\left(s_{l}, \gamma\right)$ as an example, the 2-tuples comparison operator can be introduced as follows:

(i) If $k<l$, then $\left(s_{k}, \alpha\right)$ is smaller than $\left(s_{l}, \gamma\right)$.

(ii) If $k=l$, then

i) If $\alpha=\gamma$, then $\left(s_{k}, \alpha\right)$ and $\left(s_{l}, \gamma\right)$ represent the same information.

ii) If $\alpha<\gamma$, then $\left(s_{k}, \alpha\right)$ is smaller than $\left(s_{l}, \gamma\right)$.

\subsection{Numerical scale model}

The Herrera and Martínez model is well suited to deal with uniformly and symmetrically distributed linguistic term sets. Furthermore, Dong et al. [38] extended the 2-tuples linguistic representation model to serve the linguistic term sets that are not uniformly and symmetrically distributed.

Definition 1 [38]. Let $S$ be as before, and $R$ be the set of real numbers. The function: $N S: S \rightarrow R$ is defined as a numerical scale of $S$, and $N S\left(s_{j}\right)$ is called the numerical index of $s_{j}$. If the function $N S$ is strictly monotone increasing, then $N S$ is called an ordered numerical scale.

Definition 2 [38]. Let $S, \bar{S}, N S$ on $S$ be as before. For $\left(s_{j}, \alpha\right) \in \bar{S}$, the numerical scale $N S$ on $\bar{S}$ is defined by

$$
N S\left(\left(s_{j}, \alpha\right)\right)= \begin{cases}N S\left(s_{j}\right)+\alpha\left(N S\left(s_{j+1}\right)-N S\left(s_{j}\right)\right) & \alpha \geq 0 \\ N S\left(s_{j}\right)+\alpha\left(N S\left(s_{j}\right)-N S\left(s_{j-1}\right)\right) & \alpha<0\end{cases}
$$

Clearly, the inverse function of the ordered numerical scale $N S$ exists, and is denoted as $N S^{-1}$. The function $N S^{-1}$ guarantees numerical values can be transformed into linguistic 
2-tuples.

Proposition 1 [38]. Setting $N S\left(s_{j}\right)=j(j=0,1, \ldots, G)$ yields the Herrera and Martínez model [37].

Wang and Hao [39] proposed an interesting generalized version of the Herrera and Martínez model. In the Wang and Hao model [39], the semantics of linguistic terms are defined by symmetrical trapezoidal fuzzy numbers, and the semantic of $s_{j}$ is defined by $F\left[b_{j}-\delta_{j}, b_{j}, c_{j}, c_{j}+\delta_{j}\right]$. Then, Wang and Hao [39] argued that the canonical characteristic value $(\mathrm{CCV})$ of $s_{j}$ is represented as

$$
\operatorname{CCV}\left(s_{j}\right)=\left(b_{j}+c_{j}\right) / 2 .
$$

Proposition 2 [38]. Setting $N S\left(s_{j}\right)=C C V\left(s_{j}\right)(j=0,1, \ldots, G)$ yields the Wang and Hao model [39].

Clearly, the numerical scale model presented in Dong et al. [38] proposed an integration regarding the Herrera and Martínez model [37] and the Wang and Hao model [39]. Recently, based on the research works in [38], Dong et al. [40, 41] further developed the interval numerical scales.

In this paper, we use the numerical scale model to deal with the linguistic opinions. Specifically, the numerical scales of linguistic terms are set as the CCVs of linguistic terms.

Based on numerical scales as Definition 2, Dong et al. [42] further defined balanced/ unbalanced linguistic term sets as Definition 3.

Definition 3 [42]. Let $S$ and $N S$ on $S$ be as before, and let $s^{m}$ be the middle term in $S$. Then, $S$ is a uniformly and symmetrically distributed linguistic term set if the following two conditions are satisfied:

(1) There exists an unique constant $\lambda>0$ such that $N S\left(s_{i}\right)-N S\left(s_{j}\right)=\lambda(i-j)$ for all $i, j=0,1, \ldots, G$.

(2) Let $\overline{\bar{S}}=\left\{s_{j} \mid s_{j} \in S, s_{j}>s^{m}\right\}$ and $\underline{\underline{S}}=\left\{s_{j} \mid s_{j} \in S, s_{j}<s^{m}\right\}$. Let $\#(\overline{\bar{S}})$ and $\#(\underline{\underline{S}})$ be granularity of $\overline{\bar{S}}$ and $\underline{\underline{S}}$, respectively, then $\#(\overline{\bar{S}})=\#(\underline{\underline{S}})$.

If $S$ is an uniformly and symmetrically distributed term set, then $S$ is called a balanced linguistic term set (with respect to $N S$ ). Otherwise, $S$ is called an unbalanced linguistic term set.

\subsection{The HK model}

In this paper, we adopt the HK model as the based model. If we adopt the DW model as the based model, a similar study can be proposed.

The HK model $[10,11]$ is briefly introduced as follows:

Consider a set of agents $A=\left\{a_{i} \mid i=1,2, \ldots, n\right\}$ and a set of discrete time 
$T=\{t \mid t=0,1, \ldots, N\}$. The original HK model assumes each agent expresses his/her opinion by using real number (i.e., numeric opinion). Specifically, let $Y(t)=\left(y_{1}(t), \ldots, y_{i}(t), \ldots, y_{n}(t)\right)^{T}$ be the vector of the numeric opinions of all the agents at time $t$, where $y_{i}(t)$ denotes the numeric opinion expressed by agent $a_{i}, y_{i}(t) \in[0,1]$, for $i=1,2, \ldots, n$. Let $\varepsilon_{i} \geq 0$ be the bounded confidence of agent $a_{i}$, where agent $a_{i}$ only considers the opinions which differ not more than $\varepsilon_{i}$ from his/her opinion. Therefore, the confidence set (i.e., the set of agents whose opinions are considered by agent $\left.a_{i}\right)$ is determined as

$$
I\left(a_{i}, Y(t)\right)=\left\{a_{j} \| y_{i}(t)-y_{j}(t) \mid \leq \varepsilon_{i}\right\}, i=1,2, \ldots, n,
$$

where || denotes the absolute value of a real number.

Next, each agent $a_{i}$ assigns the same weight to the agents in his/her confidence set $I\left(a_{i}, Y(t)\right)$. Specifically, let $w_{i j}(t)$ be the weight which agent $a_{i}$ assigns to $a_{j}$ at time $t$, $w_{i j}(t)$ is determined as

$$
w_{i j}(t)=\left\{\begin{array}{ll}
1 / \#\left(I\left(a_{i}, Y(t)\right)\right), & j \in I\left(a_{i}, Y(t)\right) \\
0, & j \notin I\left(a_{i}, Y(t)\right)
\end{array}, \quad i=1,2, \ldots, n,\right.
$$

where $\#(\cdot)$ denotes the number of elements in a finite set, and $w_{i j}(t) \geq 0$, such that $\sum_{j=1}^{n} w_{i j}(t)=1$.

Finally, the evolution of the opinions in the HK model is modeled as the weighted arithmetic means of opinions. Let $y_{i}(t+1)$ be the opinion of agent $a_{i}$ at time $t+1$, i.e.,

$$
y_{i}(t+1)=w_{i 1}(t) y_{1}(t)+w_{i 2}(t) y_{2}(t)+\ldots+w_{i n}(t) y_{n}(t) .
$$

If $\varepsilon_{i}=\varepsilon(i=1,2, \ldots, n)$, we call the HK model is homogeneous, otherwise heterogeneous.

\section{The proposed linguistic opinion dynamics model}

In this section we propose the LOD model, which assumes that each agent expresses and receives opinion by a linguistic simple term (i.e., linguistic opinions) at any time.

Let $A=\left\{a_{i} \mid i=1, \ldots, n\right\}$ and $T=\{t \mid t=0,1, \ldots, N\}$ be as before. Let $S=\left\{s_{j} \mid j=0,1, \ldots, G\right\}$ be a linguistic term set with odd granularity $G+1$, where the semantic of linguistic term $s_{j}$ is defined by a symmetrical trapezoidal fuzzy number $F\left[b_{j}-\delta_{j}, b_{j}, c_{j}, c_{j}+\delta_{j}\right]$. Let $O(t)=\left(o_{1}(t), o_{2}(t), \ldots, o_{n}(t)\right)$ be the vector of the linguistic opinions of all the agents at time $t$, where $o_{i}(t) \in S(i=1,2, \ldots, n)$ denotes the simple term (i.e., linguistic opinion) expressed by $a_{i}$ at time $t$.

Inspired by the original HK model, we propose the LOD model. The detailed process for LOD model can be described as follows:

Let $X(t)=\left(x_{1}(t), x_{2}(t), \ldots, x_{n}(t)\right)$ be the numerical scales vector of linguistic opinions $O(t)$, 
where $x_{i}(t)=N S\left(o_{i}(t)\right) \in[0,1]$, for $i=1,2, \ldots, n$. Without loss of generality, if $o_{i}(t)=s_{l}=$ $F\left[b_{l}-\delta_{l}, b_{l}, c_{l}, c_{l}+\delta_{l}\right]$, according to Eq. (3) then

$$
x_{i}(t)=N S\left(s_{l}\right)=C C V\left(s_{l}\right)=\left(b_{l}+c_{l}\right) / 2, \quad i=1,2, \ldots, n .
$$

Following this, let $\varepsilon_{i} \geq 0(i=1,2, \ldots, n)$ be the bounded confidence of $a_{i}$. The confidence set $\tilde{I}\left(a_{i}, X(t)\right)$ of agent $a_{i}$ in the LOD model is determined as

$$
\tilde{I}\left(a_{i}, X(t)\right)=\left\{a_{j}|| x_{i}(t)-x_{j}(t) \mid \leq \varepsilon_{i}\right\}, \quad i=1,2, \ldots, n .
$$

Next, agent $a_{i}$ assigns the same weight to the agents in his/her confidence set $\tilde{I}\left(a_{i}, X(t)\right)$. Specifically, let $\tilde{w}_{i j}(t)$ be the weight which the agent $a_{i}$ assigns to $a_{j}$ at time $t, \tilde{w}_{i j}(t)$ is determined as

$$
\widetilde{w_{i j}}(t)=\left\{\begin{array}{ll}
1 / \#\left(\tilde{I}\left(a_{i}, X(t)\right)\right), & j \in \tilde{I}\left(a_{i}, X(t)\right) \\
0, & j \notin \tilde{I}\left(a_{i}, X(t)\right)
\end{array}, \quad i=1,2, \ldots, n .\right.
$$

Let $o_{i}(t+1)$ be the linguistic opinion expressed by $a_{i}$ at time $t+1$. The evolution of the linguistic opinions is modeled as the weighted arithmetic means of linguistic opinions, i.e.,

$$
o_{i}(t+1)=\operatorname{app}\left(N S^{-1}\left(\tilde{w}_{i 1}(t) N S\left(o_{1}(t)\right)+\tilde{w}_{i 2}(t) N S\left(o_{2}(t)\right)+\ldots+\tilde{w}_{i n}(t) N S\left(o_{n}(t)\right)\right)\right), i=1, \ldots, n,(10)
$$

where $\operatorname{app}(\cdot)$ is defined as an approximate operator of the 2-tuples. For example, if $\left(s_{j}, \alpha\right) \in \bar{S}$, then $\operatorname{app}\left(s_{j}, \alpha\right)=s_{j}$.

Clearly, according to the Eqs. (7) and (9), Eq. (10) can be rewritten as:

$$
o_{i}(t+1)=\operatorname{app}\left(N S^{-1}\left(\#\left(\tilde{I}\left(a_{i}, X(t)\right)\right)^{-1} \sum_{j \in \tilde{I}\left(a_{i}, X(t)\right)} x_{j}(t)\right)\right) .
$$

Specially, if $\varepsilon_{i}=\varepsilon(i=1,2, \ldots, n)$, the LOD model is homogeneous; otherwise heterogeneous.

Note 1. Compared with the existing opinion dynamics models, the LOD model has two new features: (i) the agents express and receive linguistic opinions instead of numeric opinions; (ii) the agents' linguistic opinions are simple terms in the linguistic term sets with finite granularities at each time.

For illustrating the LOD model, we provide an example as follows:

Example 1. Assume that there are seven agents $A=\left\{a_{1}, a_{2}, a_{3}, a_{4}, a_{5}, a_{6}, a_{7}\right\}$ who express their linguistic opinions over an issue, and the linguistic opinion expressed by each agent $a_{i}(i=1, \ldots, 7)$ is a simple term in an established linguistic term set at any time. Here, without loss of generality, we use the linguistic term set in Wang and Hao [39], i.e.,

$$
S=\left\{s_{0}=I, s_{1}=E U, s_{2}=V L C, s_{3}=S C, s_{4}=I M, s_{5}=M C, s_{6}=M L, s_{7}=E L, s_{8}=C\right\},
$$

The semantics of $S$ are defined by trapezoidal fuzzy numbers in $[0,1]$ as follows: 
I Impossible $F[0,0,0,0]$.

EU Extremely unlikely $F[0,0.01,0.03,0.04]$.

$V L C \quad$ Very low chance $F[0.03,0.10,0.18,0.25]$.

SC Small chance $F[0.18,0.22,0.36,0.40]$.

IM It may $F[0.36,0.42,0.58,0.64]$.

MC Meanful chance $F[0.60,0.64,0.78,0.82]$.

ML $\quad$ Most likely $F[0.75,0.82,0.90,0.97]$.

EL Extremely likely $F[0.96,0.97,0.99,1]$.

C Certain $F[1,1,1,1]$.

Based on the above semantics, using Eq. (3) yields the corresponding numerical scales of simple terms in $S$, which are listed in Table 1.

Table 1. The numerical scales of $S$

\begin{tabular}{cccccccccc}
\hline$s_{j}$ & $j=0$ & $j=1$ & $j=2$ & $j=3$ & $j=4$ & $j=5$ & $j=6$ & $j=7$ & $j=8$ \\
\hline$N S\left(s_{j}\right)$ & 0 & 0.02 & 0.14 & 0.29 & 0.5 & 0.71 & 0.86 & 0.98 & 1 \\
\hline
\end{tabular}

Seven agents express their initial linguistic opinions $o_{i}(0)(i=1,2, \ldots, 7)$ as follows:

$O(0)=\left(o_{1}(0), o_{2}(0), o_{3}(0), o_{4}(0), o_{5}(0), o_{6}(0), o_{7}(0)\right)=\left(s_{1}, s_{3}, s_{3}, s_{4}, s_{5}, s_{6}, s_{7}\right)$.

According to Table 1, the corresponding numerical scales of the initial linguistic opinions, i.e., $\quad x_{i}(t)=N S\left(o_{i}(t)\right)(i=1,2, \ldots, 7)$, are listed in Table 2 .

Table 2. The numerical scales of the initial linguistic opinions

\begin{tabular}{cccccccc}
\hline$o_{i}(0)$ & $i=1$ & $i=2$ & $i=3$ & $i=4$ & $i=5$ & $i=6$ & $i=7$ \\
\hline$x_{i}(0)$ & 0.02 & 0.29 & 0.29 & 0.5 & 0.71 & 0.86 & 0.98 \\
\hline
\end{tabular}

Without loss of generality, let $\varepsilon=0.5$ be the homogeneous bounded confidence of agents. Then, using Eq. (8) calculates the new confidence set $\tilde{I}\left(a_{i}, X(0)\right) \quad(i=1, \ldots, 7)$ as follows:

$$
\begin{aligned}
& \tilde{I}\left(a_{1}, X(0)\right)=\{1,2,3,4\}, \tilde{I}\left(a_{2}, X(0)\right)=\{1,2,3,4,5\}, \tilde{I}\left(a_{3}, X(0)\right)=\{1,2,3,4,5\}, \\
& \tilde{I}\left(a_{4}, X(0)\right)=\{1,2,3,4,5,6,7\}, \tilde{I}\left(a_{5}, X(0)\right)=\{2,3,4,5,6,7\}, \tilde{I}\left(a_{6}, X(0)\right)=\{4,5,6,7\}, \\
& \tilde{I}\left(a_{7}, X(0)\right)=\{4,5,6,7\} .
\end{aligned}
$$

Next, using Eq. (9) calculates the weight $\tilde{w}_{i j}(0)$, for $i, j=1, \ldots, 7$. And then using Eq. (11) calculates the new linguistic opinions $o_{i}(1) \quad(i=1, \ldots, 7)$ as follows:

$$
\begin{aligned}
& o_{1}(1)=\operatorname{app}\left(N S^{-1}\left(\frac{0.02+0.29+0.29+0.5}{4}\right)\right)=\operatorname{app}\left(N S^{-1}(0.275)\right)=s_{3}, \\
& o_{2}(1)=\operatorname{app}\left(N S^{-1}\left(\frac{0.02+0.29+0.29+0.5+0.71}{5}\right)\right)=\operatorname{app}\left(N S^{-1}(0.362)\right)=s_{3}, \\
& o_{3}(1)=\operatorname{app}\left(N S^{-1}\left(\frac{0.02+0.29+0.29+0.5+0.71}{5}\right)\right)=\operatorname{app}\left(N S^{-1}(0.362)\right)=s_{3},
\end{aligned}
$$




$$
\begin{aligned}
& o_{4}(1)=\operatorname{app}\left(N S^{-1}\left(\frac{0.02+0.29+0.29+0.5+0.71+0.86+0.98}{7}\right)\right)=\operatorname{app}\left(N S^{-1}(0.521)\right)=s_{4} \\
& o_{5}(1)=\operatorname{app}\left(N S^{-1}\left(\frac{0.29+0.29+0.5+0.71+0.86+0.98}{6}\right)\right)=\operatorname{app}\left(N S^{-1}(0.605)\right)=s_{5}, \\
& o_{6}(1)=\operatorname{app}\left(N S^{-1}\left(\frac{0.5+0.71+0.86+0.98}{4}\right)\right)=\operatorname{app}\left(N S^{-1}(0.7625)\right)=s_{5}, \\
& o_{7}(1)=\operatorname{app}\left(N S^{-1}\left(\frac{0.5+0.71+0.86+0.98}{4}\right)\right)=\operatorname{app}\left(N S^{-1}(0.7625)\right)=s_{5} .
\end{aligned}
$$

We repeat the similar work as above, and obtain the linguistic opinions of agents at $t=2$, i.e., $O(2)=\left(o_{1}(2), o_{2}(2), o_{3}(2), o_{4}(2), o_{5}(2), o_{6}(2), o_{7}(2)\right)=\left(s_{4}, s_{4}, s_{4}, s_{4}, s_{4}, s_{4}, s_{4}\right)$.

Clearly, a consensus among agents at time $t=2$ has been reached.

Note 2. Recently, Wang and Mendel [43] proposed an opinion dynamics model in the linguistic environment, which is called the fuzzy opinion network (FON) model. The main differences between the LOD model and the FON model are as follows:

(i) The LOD model and the FON model are established based on the different linguistic computational models for CWW. Specifically, the LOD model is based on the 2-tuples linguistic model with numerical scales, and the FON model is based on the type-1 fuzzy sets. Specially, the membership functions of linguistic terms in the FON model are assumed to follow some specific distributions, such as the triangular distribution and the normal distribution.

(ii) The LOD model assumes that the agents' opinions are simple terms in a linguistic term set, i.e., $o_{i}(t) \in S(i=1,2, \ldots, n)$, in the dynamics of linguistic opinion formation. While the FON model assumes that the agents' opinions are the membership functions. In most of situations, these membership functions in the evolution of linguistic opinions based on the FON model cannot accurately match the semantics of the simple terms.

\section{Theoretical analysis}

In this section, we present some theoretical analyses to discuss the conditions to form a consensus or splits in the linguistic opinion formation.

Before presenting the theoretical analyses on the proposed LOD model, we introduce several definitions regarding the stabilized patterns, consensus, splits and $\varepsilon$-profile, as Definitions 4-6.

Definition 4. If the linguistic opinions satisfy $o_{i}(t)=o_{i}(t+1) \quad(i=1,2, \ldots, n)$, then the stabilized patterns in opinion formation are formed at time $t$.

Definition 5. If the stabilized patterns in opinion formation are formed at time $t$, and the linguistic opinions satisfy $o_{i}(t)=o_{i+1}(t)(i=1,2, \ldots, n-1)$, then we call a consensus among the agents is reached at time $t$; otherwise, we call the splits among the agents are formed at time $t$.

Similar to the $\varepsilon$-profile in [11], we define the $\varepsilon$-profile for the LOD model in Definition 6.

Definition 6. Let $x_{\sigma(1)}(t) \leq x_{\sigma(2)}(t) \leq \mathrm{K} \leq x_{\sigma(n)}(t)$ be a permutation of $X(t)=\left(x_{1}(t), \ldots\right.$, 
$\left.x_{n}(t)\right)$, and let $\varepsilon_{i}=\varepsilon(i=1,2, \ldots, n)$. If $x_{\sigma(i+1)}(t)-x_{\sigma(i)}(t) \leq \varepsilon \quad(i=1,2, \ldots, n-1)$, then we call $X(t)$ is a $\varepsilon$-profile at time $t$.

Let $Q$ be a finite set of discrete numbers on $R, \#(Q)$ denotes the number of elements in $Q . M_{Q}$ denotes the arithmetic mean of discrete numbers in $Q$, i.e., $M_{Q}=\#(Q)^{-1} \sum_{q \in Q} q$.

Lemma 1 [11]. Let $E$ and $Q$ be two finite sets of discrete numbers on $R$. If $\max _{e \in E} e \leq$ $\min _{q \in Q} q$, then

$$
M_{E} \leq M_{E \cup Q} \leq M_{Q} .
$$

Lemma 2 [11]. Let $B$ be a finite set of discrete numbers on $R$, and let $b_{1}, b_{2} \in B$, $B_{1}=\left\{b \in B \| b-b_{1} \mid \leq \varepsilon\right\}$ and $B_{2}=\left\{b \in B \| b-b_{2} \mid \leq \varepsilon\right\}$. If $b_{1} \leq b_{2}$, then

$$
M_{B_{1}} \leq M_{B_{2}} .
$$

Based on Lemmas 1-2, Lemma 3 is obtained.

Lemma 3. When the LOD model is homogeneous, the dynamics does not change the order of the agents' linguistic opinions, i.e., if $o_{i}(t) \leq o_{j}(t)$, then $o_{i}(t+1) \leq o_{j}(t+1)$ for all $t \in T$.

Lemma 3 shows that the sequential consistency of agents' linguistic opinions in the homogeneous LOD model is satisfied.

Theorem 1. Let $x_{\sigma(1)}(t) \leq x_{\sigma(2)}(t) \leq \mathrm{K} \leq x_{\sigma(n)}(t) \quad$ be as before, and let $\varepsilon_{i}=\varepsilon$ $(i=1,2, \ldots, n)$. If $x_{\sigma(i+1)}(t)-x_{\sigma(i)}(t)>\varepsilon(i=1,2, \ldots, n-1)$, then a consensus cannot be reached at any time $T \geq t$.

Theorem 1 shows that a consensus cannot be reached if there is a split between two agents whose opinions are adjacent in the homogeneous LOD model.

Theorem 2. When the LOD model is homogeneous, then the following statements hold:

(i) When $n=2,3$, a consensus can be reached if and only if the initial profile $X(0)$ is a $\varepsilon$-profile

(ii) When $n \geq 4$, the condition that $X(0)$ is a $\varepsilon$-profile is a necessary but not sufficient condition for reaching a consensus.

From Theorems 1 and 2, we obtain Corollary 1.

Corollary 1. When the LOD model is homogeneous, the condition that $X(0)$ is a $\varepsilon$-profile is only a necessary but not sufficient condition for reaching a consensus.

Theorem 3. Let $S$ be a balanced linguistic term set, where $N S\left(s_{j+1}\right)-N S\left(s_{j}\right)=1 / G$ $(j=0,1, \ldots, G-1)$. If $n=2, \min \left\{\varepsilon_{1}, \varepsilon_{2}\right\} \geq 1 / G$ and $\min \left\{\varepsilon_{1}, \varepsilon_{2}\right\}<\left|x_{1}(0)-x_{2}(0)\right| \leq \max \left\{\varepsilon_{1}, \varepsilon_{2}\right\}$, then a consensus can be reached after finitely many steps $m+1$, where

$$
\left\lceil\log _{2} \frac{\left|x_{1}(0)-x_{2}(0)\right|}{\min \left\{\varepsilon_{1}, \varepsilon_{2}\right\}}\right\rceil \leq m \leq\left\lceil\log _{2} \frac{G\left|x_{1}(0)-x_{2}(0)\right|-1}{G \min \left\{\varepsilon_{1}, \varepsilon_{2}\right\}-1}\right\rceil,
$$


here, $\lceil z\rceil$ denotes the smallest natural number above $z$.

\section{Simulation analysis}

In this section, we propose the simulations to explore two effects: (i) the effects of the bounded confidence on the dynamics of linguistic opinion formation, and (ii) the effects of the uniformities of linguistic term sets on the dynamics of linguistic opinion formation.

\subsection{The effects of the bounded confidence}

In this subsection, we show the effects of the bounded confidence on the dynamics of linguistic opinion formation from two aspects: (i) the effects of the bounded confidence on the sequential consistency; (ii) the effects of the bounded confidence on the consensus reaching.

\subsubsection{The effects of the bounded confidence on the sequential consistency}

In Section 4, we prove that the homogeneous LOD model satisfies the sequence consistency. In the following, we propose a counter example (i.e., Example 2) to show that the heterogeneous LOD model doesn't satisfy the sequence consistency.

Example 2. Assume that 50 agents express their linguistic opinions at time $t=0$ using simple terms of $S$ that defined in Example 1, i.e.,

$$
o_{i}(0)=\left\{\begin{array}{ll}
s_{1} & i=1,2, \ldots, 10 \\
s_{3} & i=11,12, \ldots, 20 \\
s_{4} & i=21,22, \ldots, 30 \\
s_{5} & i=31,32, \ldots, 40 \\
s_{7} & i=41,42, \ldots, 50
\end{array} .\right.
$$

According to Table 1, we can obtain the numerical scales of the initial linguistic opinions, i.e.,

$$
x_{i}(0)= \begin{cases}0.02 & i=1,2, \ldots, 10 \\ 0.29 & i=11,12, \ldots, 20 \\ 0.5 & i=21,22, \ldots, 30 \\ 0.71 & i=31,32, \ldots, 40 \\ 0.98 & i=41,42, \ldots, 50\end{cases}
$$

Meanwhile, the heterogeneous bounded confidences are given by

$$
\varepsilon_{i}= \begin{cases}0.3 & i=1,2, \ldots, 10 \\ 0.5 & i=11,12, \ldots, 20 \\ 0.2 & i=21,22, \ldots, 30 \\ 0.2 & i=31,32, \ldots, 40 \\ 0.4 & i=41,42, \ldots, 50\end{cases}
$$

Based on the values of $o_{i}(0)$ and $\varepsilon_{i}(i=1,2, \ldots, 50)$, using the LOD model obtains the evolution of the linguistic opinions, which are shown in Fig. 1. 


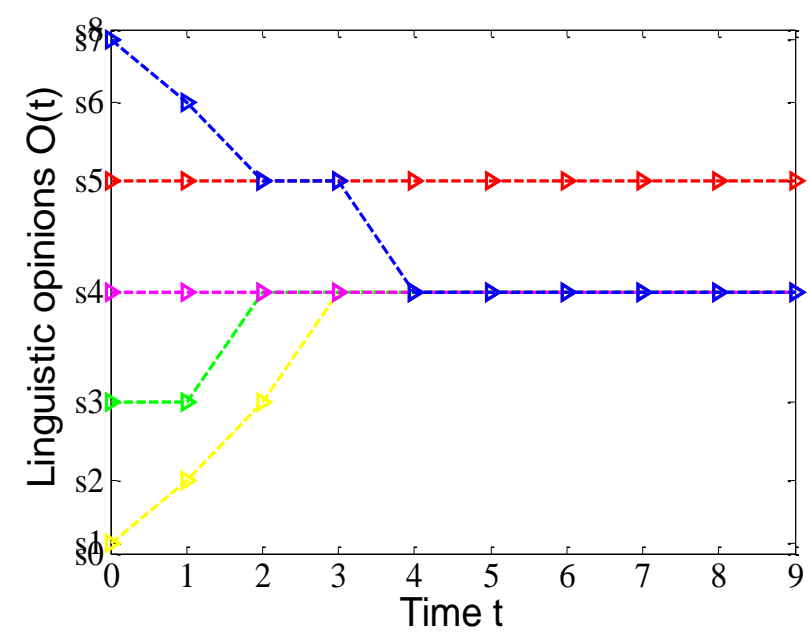

Fig. 1. The evolutions of linguistic opinions in Example 2 based on the heterogeneous LOD model

From Fig. 1, we obtain the observation: the sequence consistency of linguistic opinions holds before the time $t \leq 3$, and when $t=4$ the blue line crosses the red line. This implies that the heterogeneous LOD model doesn't satisfy the sequence consistency.

\subsubsection{The effects of the bounded confidence on the consensus reaching}

In the following, we will discuss the stabilized patterns (i.e., consensus or split) and the time for reaching a consensus (i.e., consensus time) in the homogeneous LOD model.

Let $S R$ be the flag of the stabilized result, where $S R=1$ denotes a consensus, and $S R=0$ denotes splits. Let $t^{*}$ be the time when a consensus is reached, i.e., the consensus time. Then, we present the Simulation method I to determine the $S R$ and $t^{*}$ values.

\section{Simulation method I:}

Input: $S, N S, n$ and $\varepsilon$.

Output: $S R$ and $t^{*}$.

Step 1: Let $t=0$. Linguistic opinions $o_{i}(0)(i=1,2, \ldots, n)$ are uniformly and randomly selected from $S$.

Step 2: Using Eq. (3) obtains the numerical scale values of $x_{i}(0) \quad(i=1,2, \ldots, n)$.

Step 3: Using Eqs. (8) and (11) obtains the linguistic opinions $o_{i}(t+1)(i=1,2, \ldots, n)$.

Step 4: If $o_{i}(t+1)=o_{i}(t)(i=1,2, \ldots, n)$, then go to Step 5; otherwise, let $t=t+1$, then go to Step 3 .

Step 5: If $o_{i}(t+1)=o_{i+1}(t)(i=1,2, \ldots, n-1)$, then $S R=1$ and $t^{*}=t$; otherwise, $S R=0$.

Step 6: Output $S R$ and $t^{*}$.

Here, let $S$ and $N S$ be as before that defined in Example 1. Let $n=1000$. We set different $\varepsilon$ values, and run the Simulation method I 2000 times, obtaining the average $S R$ values, which reflect the ratio of reaching a consensus in the LOD model. Fig. 2 shows the average $S R$ values under different $\varepsilon$ values. 


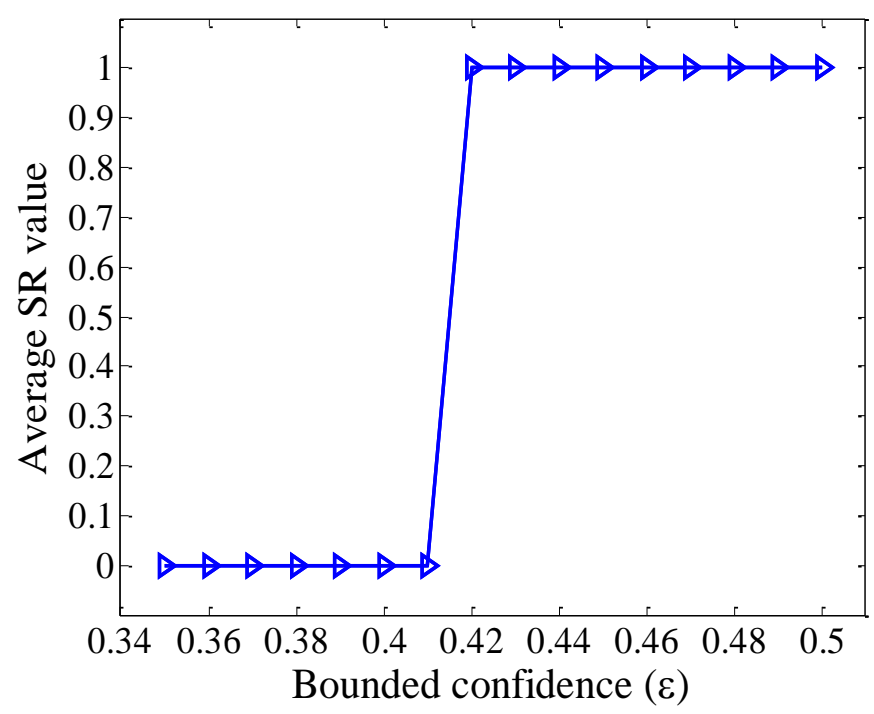

Fig. 2. The average $S R$ values under different $\varepsilon$ values

From Fig. 2, we obtain the following observations:

(i) The average $S R$ values increase as the $\varepsilon$ values increase. This implies that the opportunity for reaching a consensus will increase with the increase of the homogeneous bounded confidence.

(ii) There exists a critical point $\varepsilon_{a}$, which is called agreed confidence. When $\varepsilon \geq \varepsilon_{a}$, the average $S R$ value equals to 1 . Here, from Fig. 2, we obtain: $\varepsilon_{a}=0.42$.

Note 3.The granularities of the linguistic term sets used by agents usually are small in the practical linguistic opinion formation problems, which leads to a crisp jump at the agreed confidence $\varepsilon_{a}$ in our simulations. A consensus among the agents will be reached if the value of the bounded confidence is equal or greater than $\varepsilon_{a}$. It is worth pointing out that the crisp jump at $\varepsilon_{a}$ in our simulations will disappear when the granularities of the linguistic term sets are sufficiently large.

Note 4. In the homogeneous LOD model, setting different $S$ and $N S$ values yield different agreed confidence $\varepsilon_{a}$ values.

Moreover, we obtain the average $t^{*}$ values when $\varepsilon \geq \varepsilon_{a}=0.42$, which are shown in Fig. 3. 


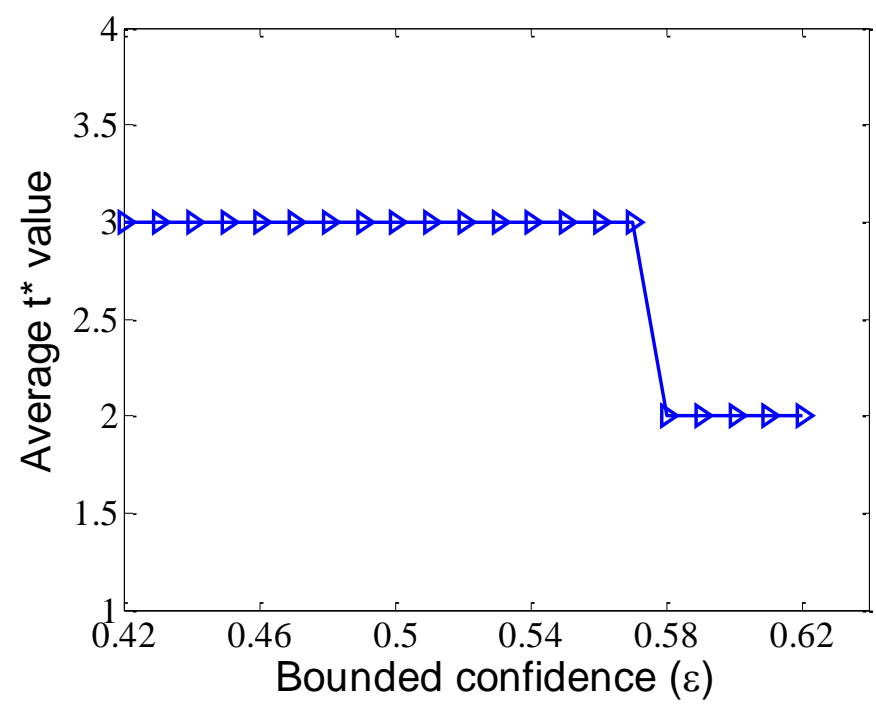

Fig. 3. The average $t^{*}$ values under different $\varepsilon \geq \varepsilon_{a}=0.42$ values

From Fig. 3, we obtain the observation: The average $t^{*}$ values decrease as the $\varepsilon$ values increase. This implies that it takes less time to reach a consensus with the increase of the homogeneous bounded confidence.

\subsection{The effects of the uniformities of the linguistic term sets}

In this subsection, based on the homogeneous LOD model, we investigate the effects of the uniformities of linguistic term sets on the consensus reaching. Specifically, when the linguistic term sets are balanced, we discuss the relationship between the granularity and the agreed confidence.

Before investigating the effects of the uniformities of linguistic term sets, we propose the measure for the uniformity of a linguistic term set as follows:

Let $S, N S$ and $G$ be as before. And let $\mu$ denote the uniformity of a linguistic term set $S$. Then, based on Definition 3, $\mu$ is calculated by

$$
\mu=1-\frac{2 \cdot \sum_{j=0}^{G}\left|N S\left(s_{j}\right)-\frac{j}{G}\right|}{G+1}
$$

Clearly, the value $\mu \in[0,1]$. And the higher value of $\mu$ indicates the higher uniformity of the linguistic term set $S$. Specially, if $\mu=1$, then $S$ is a balanced linguistic term set.

(1) The effects of the uniformities of linguistic term sets on the consensus reaching

Let $G+1=9$. Here, we set diffident numerical scale values $N S\left(s_{j}\right)$, which are shown in Table 4 (See Cases 1-5).

Table 4. The numerical scale values when $G+1=9$

\begin{tabular}{cccccccccc}
\hline$N S\left(s_{j}\right)$ & $\mathrm{j}=0$ & $\mathrm{j}=1$ & $\mathrm{j}=2$ & $\mathrm{j}=3$ & $\mathrm{j}=4$ & $\mathrm{j}=5$ & $\mathrm{j}=6$ & $\mathrm{j}=7$ & $\mathrm{j}=8$ \\
\hline Case 1 & 0 & 0.125 & 0.25 & 0.375 & 0.5 & 0.625 & 0.75 & 0.875 & 1
\end{tabular}




$\begin{array}{cccccccccc}\text { Case 2 } & 0 & 0.15 & 0.25 & 0.35 & 0.5 & 0.65 & 0.75 & 0.85 & 1 \\ \text { Case 3 } & 0 & 0.1 & 0.2 & 0.3 & 0.5 & 0.7 & 0.8 & 0.9 & 1 \\ \text { Case 4 } & 0 & 0.1 & 0.2 & 0.25 & 0.5 & 0.75 & 0.8 & 0.9 & 1 \\ \text { Case 5 } & 0 & 0.05 & 0.15 & 0.2 & 0.5 & 0.8 & 0.85 & 0.95 & 1\end{array}$

Based on Definition 3, we can easily find that only Case 1 in Table 4 denotes the numerical scale of a balanced linguistic term set. Then, according to Eq. (15), the uniformities of Cases 1-5 are calculated in Table 5 .

Table 5. The uniformities of linguistic term sets

\begin{tabular}{cccccc}
\hline & Case 1 & Case 2 & Case 3 & Case 4 & Case 5 \\
\hline$\mu$ & 1 & 0.978 & 0.933 & 0.911 & 0.844 \\
\hline
\end{tabular}

Clearly, the value of $\mu$ decreases from Case 1 to Case 5. This indicates the uniformities of linguistic term sets decrease from Case 1 to Case 5 .

Next, based on the different numerical scale values $N S$ in Cases 1-5, we run the Simulation method I 2000 times under different values for $\varepsilon$ and $N S$, obtaining the average $S R$ values, which are shown in Fig. 4.

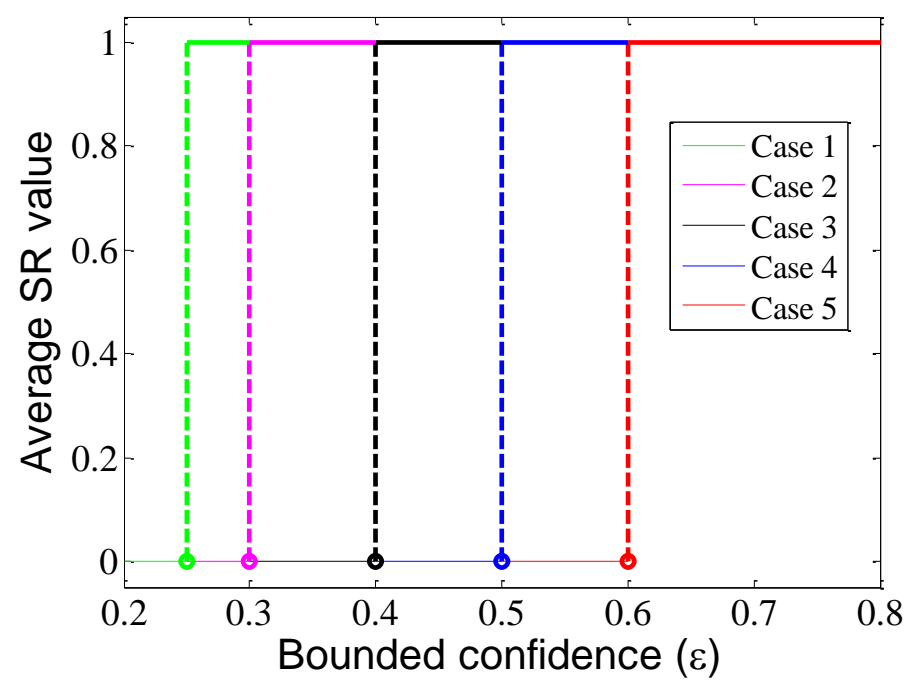

Fig. 4. The average $S R$ values associated with different $\varepsilon$ and $N S$

From Fig. 4, we obtain the observations:

(i) For all cases $1-5$, the average $S R$ values increase as the $\varepsilon$ values increase. This implies that: Regardless of the uniformities of linguistic term sets, the effect, which the increase of the bounded confidence will promote the consensus reaching, still holds.

(ii) For all cases 1-5, the $\varepsilon_{a}$ values increase as the $\mu$ values decrease. This implies that it is harder to reach a consensus with the decrease in the uniformities of linguistic term sets.

\section{(2) The relationship between the granularity and the agreed confidence}

Specifically, when the linguistic term sets are balanced, we will discuss the relationship between the granularity $G+1$ and the agreed confidence $\varepsilon_{a}$ in the homogeneous LOD model. 
Let $n=1000$. We consider 6 different balanced linguistic term sets with granularity $G+1=5,7,9,11,13$ and 15 , respectively. When setting different values for $\varepsilon$ and $G+1$, we run the Simulation method I 2000 times, obtaining the average $S R$ values, which are shown in Fig. 5 .

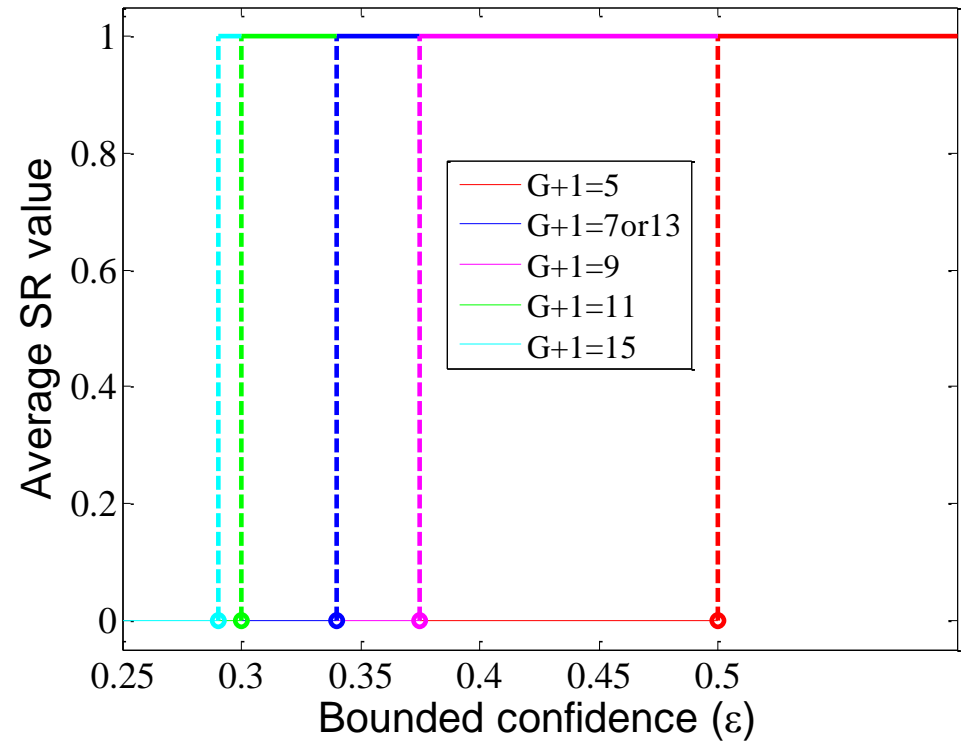

Fig. 5. The average $S R$ values associated with different $\varepsilon$ and $G+1$

Similar to the observation in Fig. 2, from Fig. 5 we obtain the following observations:

(i) The average $S R$ values increase as the $\varepsilon$ values increase for all granularities $G+1$. This implies that: regardless of the granularities of linguistic term sets, the effect, which the increase of the bounded confidence will promote the consensus reaching, still holds.

(ii) There exist different critical points under different values of $G+1$. In our simulation, we find that:

$$
\varepsilon_{a}=\left\{\begin{array}{ll}
0.5=2 / G & G+1=5 \\
0.33=2 / G & G+1=7 \\
0.375=3 / G & G+1=9 \\
0.3=3 / G & G+1=11 \\
0.33=4 / G & G+1=13 \\
0.29=4 / G & G+1=15
\end{array} .\right.
$$

In order to discuss the relationship between the granularity $G+1$ and the agreed confidence $\varepsilon_{a}$.We further investigate the agreed confidence when $17 \leq G+1 \leq 23$ by Simulation method I, which are shown as Eq. (17): 


$$
\varepsilon_{a}=\left\{\begin{array}{ll}
0.31=5 / G & G+1=17 \\
0.28=5 / G & G+1=19 \\
0.3=6 / G & G+1=21 \\
0.27=6 / G & G+1=23
\end{array} .\right.
$$

Through observing Eqs. (16)-(17), Guess 1 can be obtained.

Guess 1. Let $V \in\{0,1,2, \ldots\}$ be a set of arbitrary nonnegative integer. We guess that the relationship between the granularity $G+1$ and the agreed confidence $\varepsilon_{a}$ can be represented as Eq. (18):

$$
\varepsilon_{a}=\left\{\begin{array}{ll}
\frac{G+4}{4 G} & \text { if } G=4+4 V \\
\frac{G+2}{4 G} & \text { if } G=6+4 V
\end{array} .\right.
$$

However, it is interesting to validate this guess analytically.

\section{Conclusions}

This paper focuses on the dynamics of linguistic opinion formation problem. The main points are presented as follows:

(i) In the real-world opinion dynamics situations, agents often express and receive opinions linguistically. With this consideration, we propose a LOD model based on the bounded confidence and the linguistic 2-tuples with numerical scales.

(ii) We present some theoretical analyses to reveal the conditions of linguistic opinions to form a consensus or splits.

(iii) We design some simulations to investigate the effects of the bounded confidence and uniformities of linguistic term sets on the linguistic opinion formation, and to highlight the importance of the agree confidence in the evolution of linguistic opinions.

In this paper, we assume that the linguistic term set and the numerical scale are same for all agents. However, in the field of computing with words, due to the complexity of language comprehension, the same linguistic term may have different semantics to different agents. Therefore, it would be interesting in any future research to see the linguistic opinion dynamics model based on the linguistic term set with individual semantics is proposed.

\section{Acknowledgements}

Yucheng Dong would like to acknowledge the financial support of Grants (Nos. 71571124, 71171160) from NSF of China, and a Grant (No. xq15b01) from system science and enterprise development research center in Sichuan University.

\section{Reference}

[1] J.R.P. French, R.P. John, A formal theory of social power, Psychological Review 63 (3) (1956) 
181-194.

[2] R.A. Holley, T.M. Liggett, Ergodic theorems for weakly interacting infinite systems and the voter model, The Annals of Probability 3 (4) (1975) 643-663.

[3] R. Durrett, J.P. Gleeson, A.L. Lloyd, P.J. Mucha, F. Shi, D. Sivakoff, J.E.S. Socolarf, C. Varghese, Graph fission in an evolving voter model, Proceedings of the National Academy of Sciences (PNAS) 109 (10) (2012) 3682-3687.

[4] M.H. DeGroot, Reaching a consensus, Journal of the American Statistical Association 69 (345) (1974) 118-121.

[5] N.E. Friedkin, E.C. Johnsen, Social influence and opinions, Journal of Mathematical Sociology 15(3-4) (1990) 193-206.

[6] R. Axelrod, The dissemination of culture: A model with local convergence and global polarization, Journal of Conflict Resolution 41 (2) (1997) 203-226.

[7] V.D. Blondel, J.M. Hendrickx, J.N. Tsitsiklis, On Krause's multi-agent consensus model with state-dependent connectivity, IEEE Transactions on Automatic Control 54 (11) (2009) 2586-2597.

[8] V.D. Blondel, J.M. Hendrickx, J.N. Tsitsiklis, Continuous-time average-preserving opinion dynamics with opinion-dependent communications, SIAM Journal on Control and Optimization 48 (8) (2010) 5214-5240.

[9] G. Deffuant, D. Neau, F. Amblard, G. Weisbuch, Mixing beliefs among interacting agents, Advances in Complex Systems 3 (1) (2000) 87-98.

[10] R. Hegselmann, U. Krause, Opinion dynamics and bounded confidence models, analysis, and simulation, Journal of Artificial Societies and Social Simulation 5 (3) (2002).

[11] U. Krause, A discrete nonlinear and non-autonomous model of consensus formation, Communications in difference equations (2000) 227-236.

[12] J. Lorenz, A stabilization theorem for dynamics of continuous opinions, Physica A: Statistical Mechanics and its Applications 355 (1) (2005) 217-223.

[13] G. Weisbuch, G. Deffuant, F. Amblard, J.P. Nadal, Interacting agents and continuous opinions dynamics, Springer Berlin Heidelberg (2003) 225-242

[14] G. Weisbuch, G. Deffuant, F. Amblard, J.P. Nadal, Meet, discuss, and segregate!, Complexity 7 (3) (2002) 55-63.

[15] S.R. Etesami, T. Basar, A. Nedic, B. Touri, Termination time of multidimensional Hegselmann-Krause opinion dynamics, American Control Conference (ACC) 6 (2013) 1255-1260.

[16] S. Fortunato, V. Latora, A. Pluchino, A. Rapisarda, Vector opinion dynamics in a bounded confidence consensus model, International Journal of Modern Physics C 16 (10) (2005) 1535-1551.

[17] A. Pluchino, V. Latora, A. Rapisarda, Compromise and synchronization in opinion dynamics, The European Physical Journal B-Condensed Matter and Complex Systems 50 (1) (2006) 
169-176.

[18] G. Fu, W. Zhang, Z. Li, Opinion dynamics of modified Hegselmann-Krause model in a group-based population with heterogeneous bounded confidence, Physica A: Statistical Mechanics and its Applications 419 (1) (2015) 558-565.

[19] J. Lorenz, Heterogeneous bounds of confidence: Meet, discuss and find consensus!. Complexity 15(4) (2010) 43-52.

[20] S.M. Diao, Y. Liu, Q.A. Zeng, G.X. Luo, F. Xiong, A novel opinion dynamics model based on expanded observation ranges and individuals' social influences in social networks, Physica A: Statistical Mechanics and its Applications 415 (1) (2014) 220-228.

[21] J. Ghaderi, R. Srikant, Opinion dynamics in social networks with stubborn agents: Equilibrium and convergence rate, Automatica 50 (12) (2014) 3209-3215.

[22] H. Wang, L. Shang, R. Zhang, Opinion dynamics in networks with common-neighbors-based connections, Physica A: Statistical Mechanics and its Applications 421 (2015) 180-186.

[23] R. Hegselmann, U. Krause, Opinion dynamics driven by various ways of averaging, Computational Economics 25 (4) (2004) 381-405.

[24] I.C. Morarescu, A. Girard, Opinion dynamics with decaying confidence: application to community detection in graphs, IEEE Transactions on Automatic Control 56 (8) (2011) 1862-1873.

[25] F. Slanina, Dynamical phase transitions in Hegselmann-Krause model of opinion dynamics and consensus, The European Physical Journal B-Condensed Matter and Complex Systems 79 (1) (2011) 99-106.

[26] R. Hegselmann, U. Krause, Truth and cognitive division of labour: First steps towards a computer aided social epistemology, Journal of Artificial Societies and Social Simulation 9 (3) (2006).

[27] R. Hegselmann, S. König, S. Kurz, C. Niemann, J. Rambau, Optimal opinion control: The campaign problem, Available at SSRN 2516866, 2014.

[28] S. Kurz, Optimal Control of the Convergence Time in the Hegselmann-Krause Dynamics, Available at SSRN 2526725, 2014.

[29] X. Chen, H.J. Zhang, Y.C. Dong, The fusion process with heterogeneous preference structures in group decision making: A survey, Information Fusion 24 (2015) 72-83.

[30] Y.C. Dong, X. Chen, F. Herrera, Minimizing adjusted simple terms in the consensus reaching process with hesitant linguistic assessments in group decision making, Information Sciences, 297 (2015) 95-117.

[31] F. Herrera, S. Alonso, F. Chiclana, E. Herrera-Viedma, Computing with words in decision making: Foundations, trends and prospects, Fuzzy Optimization and Decision Making 8 (4) (2009) 337-364.

[32] L. Martínez, F. Herrera, An overview on the 2-tuple linguistic model for computing with words in decision making: Extensions, applications and challenges, Information Sciences 207 
(1) (2012) 1-18.

[33] J. Mendel, D. Wu, Perceptual Computing: Aiding people in making subjective judgments, John Wiley \& Sons, 2010.

[34] W. Pedrycz, P. Ekel, R. Parreiras, Fuzzy multicriteria decision-making: Models, methods and applications, John Wiley \& Sons, 2011.

[35] R.M. Rodríguez, L. Martínez, An analysis of symbolic linguistic computing models in decision making, International Journal of General Systems 42 (1) (2013) 121-136.

[36] G.Q. Zhang, Y.C. Dong, Y.F. Xu, Consistency and consensus measures for linguistic preference relation based on distribution assessments, Information Fusion 17 (2014) 46-55.

[37] F. Herrera, L. Martínez, A 2-tuple fuzzy linguistic representation model for computing with words, IEEE Transactions on Fuzzy Systems 8 (6) (2000) 746-752.

[38] Y.C. Dong, Y.F. Xu, S. Yu, Computing the numerical scale of the linguistic term set for the 2-tuple fuzzy linguistic representation model, IEEE Transactions on Fuzzy Systems 17 (2009) 1366-1378.

[39] J.H. Wang, J.Y. Hao, A new version of 2-tuple fuzzy linguistic representation model for computing with words, IEEE Transactions on Fuzzy Systems 14 (3) (2006) 435-445.

[40] Y.C. Dong, E. Herrera-Viedma, Consistency-driven automatic methodology to set interval numerical scales of 2-tuple linguistic term sets and its use in the linguistic GDM with preference relations, IEEE Transactions on Cybernetics 45 (2015) 780-792.

[41] Y.C. Dong, G.Q. Zhang, W.C. Hong, S. Yu, Linguistic computational model based on 2-tuples and intervals, IEEE Transactions on Fuzzy Systems 21 (2013) 1006-1018.

[42] Y.C. Dong, C.C. Li, Y. Xu, X. Gu, Consensus-based group decision making under multi-granular unbalanced 2-tuple linguistic preference relations, Group Decision and Negotiation 24 (2) (2015) 217-242.

[43] L.X. Wang, J.M. Mendel, Fuzzy opinion networks: A mathematical framework for the propagation of opinions and their uncertainties across social networks, Available at SSRN $2538553,2014$.

\section{Appendix.}

\section{The proof of Lemma 3}

If $o_{i}(t) \leq o_{j}(t)$ then $x_{i}(t) \leq x_{j}(t)$. According to Lemma 2, when the LOD model is homogeneous, let $b_{1}=x_{i}(t), b_{2}=x_{j}(t)$, then $M_{B_{1}} \leq M_{B_{2}}$. Following this,

$N S^{-1}\left(M_{B_{1}}\right) \leq N S^{-1}\left(M_{B_{2}}\right)$. As a result, $o_{i}(t+1) \leq o_{j}(t+1)$.

This completes the proof of Lemma 3.

\section{The proof of Theorem 1}

When the LOD model is homogeneous, if $x_{\sigma(i+1)}(t)-x_{\sigma(i)}(t)>\varepsilon$ at time $t$, then 
$x_{\sigma(i)}(t+1) \leq x_{\sigma(i)}(t) \quad$ and $\quad x_{\sigma(i+1)}(t+1) \geq x_{\sigma(i+1)}(t)$. Following this, $\left|x_{\sigma(i+1)}(t+1)-x_{\sigma(i)}(t+1)\right| \geq$ $\left|x_{\sigma(i+1)}(t)-x_{\sigma(i)}(t)\right|>\varepsilon$. Moreover, Lemma 3 shows that the sequential consistency of linguistic opinions can be hold in the homogeneous LOD model. Hence, the agents cannot reach a consensus at any time $T \geq t$.

This completes the proof of Theorem 1 .

\section{The proof of Theorem 2}

Necessity: According to Theorem 1, when the LOD model is homogeneous, if $X(t)=\left(x_{1}(t), x_{2}(t), \ldots, x_{n}(t)\right)$ is not a $\varepsilon$-profile at time $t$, then the agents cannot reach a consensus at any time $T \geq t$. Hence, when the LOD model is homogeneous, it is necessary that $X(0)$ is a $\varepsilon$-profile to reach a consensus among agents.

(i) When $n=2,3$, a consensus can be reached if and only if the initial profile $X(0)$ is a $\varepsilon$-profile.

\section{Sufficiency:}

i) For $n=2$, the initial profile is a $\varepsilon$-profile. Because $\left|x_{1}(0)-x_{2}(0)\right| \leq \varepsilon$, then a consensus can be reached.

ii) For $n=3$, the initial profile is a $\varepsilon$-profile. Without loss of generality, there are three cases:

Case 1: If $x_{1}(0)=x_{2}(0)=x_{3}(0)$, then it has already reached a consensus.

Case 2: If $x_{1}(0) \neq x_{2}(0)=x_{3}(0), x_{1}(0)=x_{2}(0) \neq x_{3}(0)$ or $x_{1}(0)=x_{3}(0) \neq x_{2}(0)$, because the initial profile is a $\varepsilon$-profile, then the consensus will be reached at time $t=1$.

Case 3: If $x_{1}(0) \neq x_{2}(0), \quad x_{1}(0) \neq x_{3}(0) \quad$ and $\quad x_{2}(0) \neq x_{3}(0)$, we assume that $x_{1}(0)<x_{2}(0)<x_{3}(0)$. Without loss of generality, there are two subcases:

Case A: The homogeneous LOD model with a balanced linguistic term set.

The numerical scales of the balanced linguistic term set satisfy that $N S\left(s_{j+1}\right)-N S\left(s_{j}\right)=\frac{1}{G}$ $(j=0,1, \ldots, G)$. Following this, let $x_{3}(0)-x_{2}(0)=\frac{\zeta_{1}}{G}$ and $x_{2}(0)-x_{1}(0)=\frac{\zeta_{2}}{G}$, where $\zeta_{1}$ and $\zeta_{2}$ are two positive integers.

Let $D(t)=x_{3}(t)-x_{1}(t)$, if $D(0)=x_{3}(0)-x_{1}(0) \leq \varepsilon$, then a consensus has been reached. Otherwise, there are four subcases for $D(1)$ :

Case A1: If both $\zeta_{1}$ and $\zeta_{2}$ are even numbers, then $x_{3}(1) \leq \frac{x_{2}(0)+x_{3}(0)}{2}$ and $x_{1}(1) \geq \frac{x_{1}(0)+x_{2}(0)}{2}$. Hence 


$$
\begin{aligned}
D(1) & \leq \frac{x_{2}(0)+x_{3}(0)}{2}-\frac{x_{1}(0)+x_{2}(0)}{2} \\
& =\frac{x_{3}(0)-x_{1}(0)}{2} \leq \varepsilon
\end{aligned}
$$

Case A2: If $\zeta_{1}$ is a even number and $\zeta_{2}$ is an odd number, then $x_{3}(1) \leq \frac{x_{2}(0)+x_{3}(0)}{2}$ and $x_{1}(1) \geq \frac{x_{1}(0)+x_{2}(0)}{2}+\frac{1}{2 G}$. Hence

$$
\begin{aligned}
D(1) & \leq \frac{x_{2}(0)+x_{3}(0)}{2}-\left(\frac{x_{1}(0)+x_{2}(0)}{2}+\frac{1}{2 G}\right) \\
& =\frac{x_{3}(0)-x_{1}(0)}{2}-\frac{1}{2 G}<\varepsilon
\end{aligned}
$$

Case A3: If both $\zeta_{1}$ and $\zeta_{2}$ are odd numbers, then $x_{3}(1) \leq \frac{x_{2}(0)+x_{3}(0)}{2}+\frac{1}{2 G}$ and $x_{1}(1) \geq \frac{x_{1}(0)+x_{2}(0)}{2}+\frac{1}{2 G}$. Hence

$$
\begin{aligned}
D(1) & \leq \frac{x_{2}(0)+x_{3}(0)}{2}+\frac{1}{2 G}-\left(\frac{x_{1}(0)+x_{2}(0)}{2}+\frac{1}{2 G}\right) \\
& =\frac{x_{3}(0)-x_{1}(0)}{2}<\varepsilon
\end{aligned}
$$

Case A4: If $\zeta_{1}$ is a even number and $\zeta_{2}$ is an odd number, then $x_{3}(1) \leq \frac{x_{2}(0)+x_{3}(0)}{2}+$

$$
\begin{aligned}
\frac{1}{2 G} \text { and } & x_{1}(1) \geq \frac{x_{1}(0)+x_{2}(0)}{2} . \text { Hence } \\
D(1) & \leq \frac{x_{2}(0)+x_{3}(0)}{2}+\frac{1}{2 G}-\frac{x_{1}(0)+x_{2}(0)}{2} \\
& =\frac{x_{3}(0)-x_{1}(0)}{2}+\frac{1}{2 G}<\frac{1}{2}\left(2 \varepsilon-\frac{1}{G}\right)+\frac{1}{2 G} \leq \varepsilon .
\end{aligned}
$$

This implies $D(2)=0$, i.e., a consensus can be reached at $t=2$.

Case B: The homogeneous LOD model with an unbalanced linguistic term set.

We assume that $x_{1}(0)<x_{2}(0)<x_{3}(0) \quad$ and $\quad x_{2}(0)-x_{1}(0)<x_{3}(0)-x_{2}(0)$. Let $D(t)=x_{3}(t)-x_{1}(t)$, if $D(0)=x_{3}(0)-x_{1}(0) \leq \varepsilon$ then a consensus has been reached. Otherwise, because

$$
x_{1}(t) \geq \frac{x_{1}(t-1)+x_{2}(t-1)}{2}
$$

and

$$
x_{3}(t) \leq x_{3}(t-1),
$$

then 


$$
\begin{aligned}
D(t) & \leq x_{3}(t-1)-\frac{x_{1}(t-1)+x_{2}(t-1)}{2} \\
& =\frac{1}{2}\left(x_{3}(t-1)-x_{2}(t-1)\right)+\frac{1}{2}\left(x_{3}(t-1)-x_{1}(t-1)\right) \\
& \leq \frac{1}{2}\left(x_{3}(t-2)-x_{2}(t-2)\right)+\frac{1}{2}\left(x_{3}(t-2)-\frac{x_{1}(t-2)+x_{2}(t-2)}{2}\right) \\
& =\frac{3}{4}\left(x_{3}(t-2)-x_{2}(t-2)\right)+\frac{1}{4}\left(x_{3}(t-2)-x_{1}(t-2)\right) \\
& \leq \frac{3}{4}\left(x_{3}(t-3)-x_{2}(t-3)\right)+\frac{1}{4}\left(x_{3}(t-3)-\frac{x_{1}(t-3)+x_{2}(t-3)}{2}\right) \\
& =\frac{7}{8}\left(x_{3}(t-3)-x_{2}(t-3)\right)+\frac{1}{8}\left(x_{3}(t-3)-x_{1}(t-3)\right) \\
& \leq \frac{15}{16}\left(x_{3}(t-4)-x_{2}(t-4)\right)+\frac{1}{16}\left(x_{3}(t-4)-x_{1}(t-4)\right) \\
& =\left(x_{3}(0)-x_{2}(0)\right)+\frac{1}{2^{t}}\left(x_{2}(0)-x_{1}(0)\right) \\
\ldots . . & \leq \frac{2^{t}-1}{2^{t}}\left(x_{3}(0)-x_{2}(0)\right)+\frac{1}{2^{t}}\left(x_{3}(0)-x_{1}(0)\right) \\
& \leq \frac{2^{k}-1}{2^{k}}\left(x_{3}(t-k)-x_{2}(t-k)\right)+\frac{1}{2^{k}}\left(x_{3}(t-k)-x_{1}(t-k)\right) \\
\ldots . . &
\end{aligned}
$$

Since $\lim _{t \rightarrow \infty} \frac{1}{2^{t}}\left(x_{2}(0)-x_{1}(0)\right)=0$, then $D(t) \leq x_{3}(0)-x_{2}(0) \leq \varepsilon$. This implies $D(t+1)=0$.

Hence, when the LOD model is homogeneous, if the initial profile is a $\varepsilon$-profile, then a consensus can be reached in the case of $n=3$.

(ii) When $n \geq 4$, the condition that $X(0)$ is a $\varepsilon$-profile is only a necessary but not sufficient condition for reaching a consensus.

In the following, we propose two counter example (i.e., Cases $\mathrm{A}$ and $\mathrm{B}$ ) to show that $X(0)$ is a $\varepsilon$-profile is not a sufficient condition for reaching a consensus when $n \geq 4$.

Case A: The homogeneous LOD model with a balanced linguistic term set.

Suppose the numerical scales of a balanced linguistic term set with granularity $G+1$ are as follows: $1 / G, 2 / G, 3 / G, \ldots,(G-1) / G, 1$.

Setting $\varepsilon=1 / G$. Following this, the initial profile is given by $x_{i}(0)=i / G$ for $1 \leq i \leq 3$ and $x_{i}(0)=4 / G$ for $4 \leq i \leq n$. Clearly, $X(0)$ is a $\varepsilon$-profile.

When $t=1$ one obtains $x_{1}(1)=2 / G, x_{2}(1)=2 / G$,

$$
x_{3}(1)=N S\left(\operatorname{app}\left(N S^{-1}\left(\frac{2 / G+3 / G+4(n-3) / G}{n-1}\right)\right)\right)
$$




$$
\begin{aligned}
& =N S\left(\operatorname{app}\left(N S^{-1}\left(\frac{4 n-7}{G(n-1)}\right)\right)\right) \\
& = \begin{cases}\frac{3}{G}, & n=4,5,6 \\
\frac{4}{G}, & n \geq 7\end{cases}
\end{aligned}
$$

and $x_{i}(1)=4 / G$ for $4 \leq i \leq n$. There are two subcases:

Case A1: If $n \geq 7$, then $x_{3}(1)=4 / G$. As a result, $x_{3}(1)-x_{2}(1)=2 / G>\varepsilon=1 / G$. According to Theorem 1, a consensus among agents cannot be reached.

Case A2: If $n=4,5,6, x_{3}(1)=3 / G$.

When $t \geq 2$, it remains that $x_{1}(t)=2 / G, x_{2}(t)=2 / G, x_{3}(t)=2 / G$ and $x_{i}(t)=4 / G$ for $4 \leq i \leq n$. Here, $X(t)$ is a $\varepsilon$-profile for all $t \in T$, but a consensus among agents cannot be reached.

Case B: The homogeneous LOD model with an unbalanced the linguistic term set.

Suppose the numerical scales of an unbalanced linguistic term set with granularity 9 are as follows: $0,0.1,0.2,0.3,0.5,0.7,0.8,0.9,1$.

Setting $\varepsilon=0.2$. Following this, the initial profile is given by $x_{1}(0)=0.1, x_{2}(0)=0.2$, $x_{3}(0)=0.3$ and $x_{i}(0)=0.5$ for $4 \leq i \leq n$. Obviously, $X(0)$ is a $\varepsilon$-profile.

When $t=1$ one obtains $x_{1}(1)=0.2, \quad x_{2}(1)=0.2$,

$$
\begin{aligned}
x_{3}(1) & =N S\left(\operatorname{app}\left(N S^{-1}\left(\frac{0.1+0.2+0.3+0.5 \times(n-3)}{n}\right)\right)\right) \\
& =N S\left(\operatorname{app}\left(N S^{-1}\left(0.5-\frac{0.9}{n}\right)\right)\right) \\
& = \begin{cases}0.3, & n=4,5,6,7,8 \\
0.5, & n \geq 9\end{cases}
\end{aligned}
$$

and $x_{i}(1)=0.5$ for $4 \leq i \leq n$. There are two subcases:

Case B1: If $n \geq 9$ then $x_{3}(1)=0.5$. As a result, $x_{3}(1)-x_{2}(1)=0.3>\varepsilon=0.2$. According to Theorem 1, a consensus among agents cannot be reached.

Case B2: If $n=8$ then $x_{3}(1)=0.3$, but when $t=2$ one obtains $x_{1}(2)=0.2, x_{2}(2)=0.2$, $x_{3}(2)=0.5$ and $x_{i}(2)=0.5$ for $4 \leq i \leq n$. As a result, $x_{3}(2)-x_{2}(2)=0.3>\varepsilon=0.2$. According to Theorem 1, a consensus among agents cannot be reached.

Case B3: If $n=4,5,6,7$, then $x_{3}(1)=0.3$. However, when $t \geq 2$ it remains that $x_{1}(t)=0.2, \quad x_{2}(t)=0.2, \quad x_{3}(t)=0.3 \quad$ and $\quad x_{i}(t)=0.5$ for $4 \leq i \leq n$. Here, $X(t)$ is a $\varepsilon$-profile for all $t \in T$, but a consensus among agents cannot be reached.

Hence, when $n \geq 4$ the condition that $X(0)$ is a $\varepsilon$-profile is only a necessary but not 
sufficient condition for reaching a consensus.

This completes the proof of Theorem 2.

\section{The proof of Theorem 3}

Assume that $x_{1}(0) \leq x_{2}(0), \varepsilon_{1} \leq \varepsilon_{2}$ and $x_{2}(0)-x_{1}(0)=\frac{\zeta_{3}}{G}$, where $\zeta_{3}$ is a positive integer. When $\varepsilon_{1}=\min \left\{\varepsilon_{1}, \varepsilon_{2}\right\}<x_{1}(0)-x_{2}(0) \mid \leq \max \left\{\varepsilon_{1}, \varepsilon_{2}\right\}=\varepsilon_{2}$, we consider two cases:

Case 1: $\zeta_{3}$ is a even number for all $t \in T$.

There exists a minimum steps $m_{l}$ such that $\left|x_{1}\left(m_{l}\right)-x_{2}\left(m_{l}\right)\right| \leq \varepsilon_{1}$ and the consensus is reached after $m_{l}+1$ steps, where $x_{1}(t)=x_{1}(0) \quad$ and $\quad x_{2}(t)=\frac{1}{2}\left(x_{1}(t-1)+x_{2}(t-1)\right) \quad$ for all $t \leq m_{l}$. Hence, $\quad\left|x_{1}(t)-x_{2}(t)\right|=\frac{1}{2}\left|x_{1}(t-1)-x_{2}(t-1)\right|$ for all $t \leq m_{l}$. Induction yields $\left|x_{1}\left(m_{l}\right)-x_{2}\left(m_{l}\right)\right|=\frac{1}{2^{m_{l}}}\left|x_{1}(0)-x_{2}(0)\right| \quad$. Therefore, $\quad \frac{1}{2^{m_{l}}}\left|x_{1}(0)-x_{2}(0)\right| \leq \varepsilon_{1} \quad$ and $\quad$ it $\quad$ follows $m_{l} \geq \log _{2} \frac{\left|x_{1}(0)-x_{2}(0)\right|}{\varepsilon_{1}}$.

Case 2: $\zeta_{3}$ is an odd number for all $t \in T$.

Then there exists a maximum steps $m_{h}$ such that $\left|x_{1}\left(m_{h}\right)-x_{2}\left(m_{h}\right)\right| \leq \varepsilon_{1}$ and the consensus is reached after $m_{h}+1$ steps, where $x_{1}(t)=x_{1}(0)$ and $x_{2}(t)=\frac{1}{2}\left(x_{1}(t-1)+x_{2}(t-1)\right)+\frac{1}{2 G}$ for all $t \leq m_{h}$.

When $t=1,\left|x_{1}(1)-x_{2}(1)\right|=\frac{1}{2}\left|x_{1}(0)-x_{2}(0)-\frac{1}{G}\right| \leq \frac{1}{2}\left|x_{1}(0)-x_{2}(0)\right|+\frac{1}{2 G}$, and when $t=2$,

$$
\begin{aligned}
\left|x_{1}(2)-x_{2}(2)\right| & =\frac{1}{2}\left|x_{1}(1)-x_{2}(1)-\frac{1}{G}\right| \leq \frac{1}{2}\left|x_{1}(1)-x_{2}(1)\right|+\frac{1}{2 G} \\
& \leq \frac{1}{2^{2}}\left|x_{1}(0)-x_{2}(0)\right|+\frac{1}{2^{2} G}+\frac{1}{2 G}
\end{aligned}
$$

until $t=m_{h}$,

$$
\begin{aligned}
\mid x_{1}\left(m_{h}\right)-x_{2}\left(m_{h}\right) & \leq \frac{1}{2^{m_{h}}}\left|x_{1}(0)-x_{2}(0)\right|+\frac{1}{2^{m_{h}} G}+\cdots+\frac{1}{2^{2} G}+\frac{1}{2 G} \\
& =\frac{1}{2^{m_{h}}}\left|x_{1}(0)-x_{2}(0)\right|+\frac{1}{G}\left(1-\frac{1}{2^{m_{h}}}\right)
\end{aligned}
$$

If $\left|x_{1}\left(m_{h}\right)-x_{2}\left(m_{h}\right)\right| \leq \varepsilon_{1}$, then $\frac{1}{2^{m_{h}}}\left|x_{1}(0)-x_{2}(0)\right|+\frac{1}{G}\left(1-\frac{1}{2^{m_{h}}}\right) \leq \varepsilon_{1}$, and it follows $m_{h} \geq \log _{2} \frac{G\left|x_{1}(0)-x_{2}(0)\right|-1}{G \varepsilon_{1}-1}$. 
Without loss of generality, it satisfies

$$
\left\lceil\log _{2} \frac{\left|x_{1}(0)-x_{2}(0)\right|}{\min \left\{\varepsilon_{1}, \varepsilon_{2}\right\}}\right\rceil \leq m\left\lceil\log _{2} \frac{G\left|x_{1}(0)-x_{2}(0)\right|-1}{G \min \left\{\varepsilon_{1}, \varepsilon_{2}\right\}}\right\rceil .
$$

This completes the proof of Theorem 3 . 